\title{
Patterns of home- and community-based services in older adults with dementia: an analysis of the long-term care system in Taiwan
}

Wen-Fu Wang ${ }^{1,2}$, Yung-Yu Su ${ }^{3}$, Kai-Ming Jhang ${ }^{1}$ and Chun-Min Chen ${ }^{4^{*}}$

\begin{abstract}
Background: Understanding the specific characteristics of the patients with dementia is essential in developing services required to meet their needs. The purpose of this study was to investigate the patterns of use of homeand community-based services (HCBS) by patients with dementia and the factors influencing the utilisation of these services.

Methods: We analysed a dataset of patients with dementia receiving long-term care at a medical centre. All participating patients were required to complete a structured interview form every 6 months to assess their need for service utilisation. Between 2015 and 2018, a total of 822 patients fulfilled the criteria for dementia, and 737 people had assessment records, of whom 244 had used social services. Robust Poisson regression analyses were performed to estimate HCBS usage and the factors influencing service utilisation.

Results: The overall service utilisation rate was $33 \%$. Most patients used only one service, and assistive devices were used as the main service. Regarding the factors influencing service use, dementia concomitant with dependency increased the use of HCBS. These results suggest that patients with mild dependency might prefer to use community support services, whereas those with moderate to severe dependency being more likely to utilise in-home care services.

Conclusions: This study provides empirical evidence regarding the use of long-term care resources by patients with dementia in the community. Providing customised HCBS, rather than a non-specialised service assumed to be suitable for every patient, is essential for ensuring good patient care. In addition, attention needs to be paid to patients with care needs who do not use HCBS.
\end{abstract}

Keywords: Dementia, Long-term care, HCBS, Utilisation, Disability

\footnotetext{
* Correspondence: cmc202@gmail.com

${ }^{4}$ Big Data Center, Changhua Christian Hospital, No. 135, Nanhsiao Street, 500-06 Changhua City, Changhua County, Taiwan

Full list of author information is available at the end of the article
}

(C) The Author(s). 2021 Open Access This article is licensed under a Creative Commons Attribution 4.0 International License, which permits use, sharing, adaptation, distribution and reproduction in any medium or format, as long as you give appropriate credit to the original author(s) and the source, provide a link to the Creative Commons licence, and indicate if changes were made. The images or other third party material in this article are included in the article's Creative Commons. licence, unless indicated otherwise in a credit line to the material. If material is not included in the article's Creative Commons licence and your intended use is not permitted by statutory regulation or exceeds the permitted use, you will need to obtain permission directly from the copyright holder. To view a copy of this licence, visit http://creativecommons.org/licenses/by/4.0/ The Creative Commons Public Domain Dedication waiver (http://creativecommons.org/publicdomain/zero/1.0/) applies to the data made available in this article, unless otherwise stated in a credit line to the data. 


\section{Background}

Population ageing is one of the major issues affecting all developed countries including Taiwan, which is a social time bomb due to its ageing population. The proportion of the population aged 65 years and above in Taiwan has doubled from $7 \%$ to 1993 to $14 \%$ in 2018 [1], making it an 'aged society' according to the World Health Organization. Dementia is a major public health issue in an ageing population. In Taiwan, there are 3.6 million people aged 65 and above, among whom 280,783 have dementia. This implies that for every 12 elderly Taiwanese, there is one person with dementia [2]. Such a high incidence of this disease has a great impact on family life and society. In the modern society, two family members often work full time, and with the prevalence of nuclear families, the demand for long-term care is rising.

Long-term care (LTC) policies are being implemented worldwide in response to the ageing trend [3]. According to the World Bank, LTC financing can be classified into four systems: social insurance models (applied in Netherlands, Germany, Japan, and the Republic of Korea), the universal model (applied in Denmark, Finland and Sweden), means-tested systems (applied in the United Kingdom and the United States), and hybrid systems and approaches (applied in France) [4]. LTC financing in Taiwan is similar to the universal model (taxbased universal public LTC coverage), although a national LTC insurance system can also be built once the development of the service system is complete [5].

Providing care to the rising number of people with dementia remains a public health challenge for health systems worldwide. To tackle these issues, the Taiwanese government has devised a series of LTC-related policies, including the LTC 10-year plan version 1 (LTC 1.0) launched in 2007, and the reformed LTC plan version 2.0, launched in 2016 [6]. LTC 2.0 expanded the community-based and institutional residential dementia care resources already stated by the LTC 1.0 to include dementia patients over 50 years of age as a service target. Moreover, LTC 2.0 also proposed the creation of integrated dementia day care centres that connect services and potential users through case management. The use of different types of care offered to people with dementia may have changed due to the roll-out of the LTC 2.0 reforms, with a lack of empirical evidence regarding the actual use of these services.

Home- and community-based services (HCBS) and support are being increasingly recognised as key components of high-quality dementia care [7]. HCBS is designed to supplement the inadequacy of informal care and assist with the care of home-bound or low-income elderly people [8]. Although the usage of HCBS by older adults who require LTC has been studied [9], service use might be influenced by differences in healthcare delivery systems and cultures $[9,10]$. Frequent challenges encountered by people with dementia and their caregivers in using HCBS include limited awareness of dementia and lack of information about available services [11, 12]. Previous studies on dementia care have mainly focused on the use of healthcare service and the cost of these services, as the primary resource of analysing utilisation outcomes $[13,14]$. To our knowledge, no study has explored the patterns of HCBS usage by elderly patients with dementia in Taiwan. Empirical knowledge of HCBS utilisation is essential to avoid insufficient and inefficient policies. Hence, examining and understanding their current use is essential.

The purpose of this study was to explore the use of HCBS and the factors influencing its usage from the perspective of patients with dementia in Changhua City, Taiwan. This study attempted to resolve the current information gap by (1) examining the patterns of usage of HCBS provided for LTC, (2) determining the frequency and combinations of HCBS utilisation, and (3) identifying the important factors affecting the use of these services. Understanding HCBS utilisation, including the frequency and combination of services used is necessary to inform about the care needs of the dementia population, such as needs specific to dementia subtypes, as well as for guiding LTC resource allocation.

\section{Methods}

\section{Data source and study design}

This retrospective cross-sectional study used data from a self-developed, integrated dementia care programme, a database from the Changhua Christian Hospital $(\mathrm{CCH})$, maintained by the Changhua Christian Hospital, and Ministry of Health and Welfare in Taiwan. Diagnostic and Statistical Manual of Mental Disorders (DSM-V) criteria were used to diagnose patients with dementia [15]. All assessments were completed by an integrated team, which included physicians, psychologists, social workers, dieticians, occupational therapists, pharmacists, and nursing case managers. Patients diagnosed with dementia were recruited as the study subjects for analysis. After the diagnosis of dementia, the team conducted face-to-face interviews with community residents and their care partners, and after evaluation of each case, a personalised care plan was devised. Follow-up telephonic interviews were conducted mainly by the nursing case manager. If the participants were unable to answer questions independently, a family member provided them with assistance or answered on their behalf. The assessment and follow-up interview processes have been previously described [16].

\section{Ethical considerations}

This study being a retrospective chart review, the teambased assessment information was used for analysis, and 
informed consent was not obtained since this study used de-identified data with secondary data analysis. The study protocol was approved by the Human Research Protection Program of Changhua Christian Hospital and adhered to the principles of the Declaration of Helsinki (approval no.: 160615; 200128).

\section{Settings and participants}

In this study, we analysed a LTC dataset containing records of patients with dementia living in a central city, in one of Taiwan's six metropolises. The records contain details of the assessment initially needed and reassessments by telephone interviews every 6 months. During this follow-up process, health indicators and service usage of the care recipient were recorded. The original study was of an open cohort design, and as of September 2018, the research dataset contained records of 822 dementia patients who underwent preliminary evaluations during 2015-2018. The data was cleaned to exclude patients who did not attend reassessments of HCBS use by telephone $(n=38)$ and those living in care facilities $(n=47)$, leaving a total of 737 patients who met the study criteria. Out of the 737 selected patients, 244 patients had used social services during the follow-up period. It was determined from the telephone assessment process that these dementia patients had used HCBS after diagnosis.

\section{Measurements \\ Independent Variables}

In the LTC dataset, case assessment records included baseline sociodemographic variables, and physiological and psychological functions. Participants' age, sex, marital status, and caregiver were recorded as demographic data. Physiological functions were evaluated according to hypnotic use (yes or no), cardiovascular disease (yes or no), cerebrovascular accident (yes or no), and dependency status. Dependency status was assessed using the International Classification of Functioning (ICF)-based measurement of functioning ( 0 - no problem, 1 - mild problem, 2 - moderate problem, 3 - severe problem, 4 - complete problem) [17]. Psychological functions were determined on the basis of an affirmative response (yes) to emotional, behavioural, or psychological questions regarding whether the patient had any one of the following conditions during assessment with patients and their caregivers: emotional problems (i.e. crying, fear, dysthymic, depressive, anxiety, anger, emotional liability, apathy, worry, fidgety), or behavioural or psychological problems (agitation, akathisia, wandering, curse, shadowing, aggression, akinetic, stereotype, abnormal circadian rhythm).

\section{Outcome Variables}

HCBS data were collected from the cohort entry date to the end of 2018, and information on service usage was reported during the need assessment [16]. HCBS resources included the following: home services (such as laundry, housework, and kitchen-based tasks); respite care (in-home, out-of-home, and overnight care); home nursing care; community care centres; home- and communitybased rehabilitation; assistive devices; adult day care; adult foster care; home meal delivery; transportation services; mobile shower/bath services; support care for caregivers (social activities such as lectures, outreach, consultation, relief travel, and fraternity); barrier-free environments; and 'School of Wisdom' (group cognitive training courses). The use of each service was categorised, with service use set to 1 (used) or 0 (not used). The amount of service usage for an individual was calculated based on the recorded resources (the same service was calculated only once). The scope of service usage was scored from to 114; the higher the score, the more services were used.

\section{Data analyses}

Descriptive analyses (mean, frequencies, and percentages) were conducted on demographic and clinical data to characterise the sample. A t-test was conducted to compare mean age. The relationships between categorical variables were assessed using the chi-square test. Patients who used $\geq 1$ resource were compared to non-users (Table 1). The service use patterns are shown in Fig. 1. The goal of this study was to analyse the different individual effects of independent variables on one dependent variable, the ultimate dependent variable of interest being the count of usage of social services. As it is a count variable and not normally distributed, the Poisson regression model [18, 19] was employed to estimate the number of HCBS used (discrete count outcomes) by patients with dementia. Since the variance (0.478) and the mean (0.420) are equal, implying that the dispersion statistic equals one, a robust Poisson model was used to estimate the regression parameters. Since the service observation period for each participant was different, we added the number of telephone visits (reassessment) in the study period as an offset variable to adjust for total service usage[18-20] (Table 2). We further incorporated service use as service combinations to assess whether service usage was different at different levels of dependency (Fig. 2). IBM SPSS Statistics for Windows, version 22.0 (IBM Corporation, Armonk, NY, USA) was used to analyse the results. The level of significance was set at $p<0.05$.

\section{Results}

\section{Basic information on Social Services Utilisation}

Of the 244 HCBS users, $55.3 \%$ of the patients with dementia used services within 365 days after the initial diagnosis, and the remaining (44.7\%) started to use services after 1 year (data not shown). The results shown in Table 1 indicate that most patients with dementia were older than 
Table 1 Characteristics of the study group by service use $(N=737)$

\begin{tabular}{|c|c|c|c|c|c|c|}
\hline & & Health $c$ & jervice & & & \\
\hline & & Non-use & & Users & & $p$ value \\
\hline & & $n=493$ & $\%$ & $n=244$ & $\%$ & \\
\hline Age (years) & mean $\pm s d$ & 77.88 & \pm 8.52 & 81.41 & \pm 7.81 & $* * *$ \\
\hline Age & $<=70$ & 90 & $18 \%$ & 18 & $7 \%$ & $* * *$ \\
\hline & $71-80$ & 166 & $34 \%$ & 73 & $30 \%$ & \\
\hline & $\geq 81$ & 237 & $48 \%$ & 153 & $63 \%$ & \\
\hline Sex & female & 320 & $65 \%$ & 151 & $62 \%$ & 0.421 \\
\hline & male & 173 & $35 \%$ & 93 & $38 \%$ & \\
\hline Marital status & married & 288 & $58 \%$ & 127 & $52 \%$ & 0.101 \\
\hline & divorced/widowed/separated & 205 & $42 \%$ & 117 & $48 \%$ & \\
\hline Caregiver & myself & 45 & $9 \%$ & 17 & $7 \%$ & 0.114 \\
\hline & spouse/partner & 167 & $34 \%$ & 70 & $29 \%$ & \\
\hline & children & 151 & $31 \%$ & 72 & $30 \%$ & \\
\hline & relatives/friends & 58 & $12 \%$ & 32 & $13 \%$ & \\
\hline & foreign worker/nursing care & 72 & $15 \%$ & 53 & $22 \%$ & \\
\hline Hypnotics & no & 384 & $78 \%$ & 161 & $66 \%$ & ** \\
\hline & yes & 109 & $22 \%$ & 83 & $34 \%$ & \\
\hline CVD & no & 430 & $87 \%$ & 211 & $86 \%$ & 0.880 \\
\hline & yes & 47 & $10 \%$ & 24 & $10 \%$ & \\
\hline CVA & no & 422 & $86 \%$ & 191 & $78 \%$ & $* *$ \\
\hline & yes & 55 & $11 \%$ & 44 & $18 \%$ & \\
\hline Dependency status & without ICF problems & 338 & $69 \%$ & 94 & $39 \%$ & $* * *$ \\
\hline & mild & 91 & $18 \%$ & 52 & $21 \%$ & \\
\hline & moderate & 38 & $8 \%$ & 57 & $23 \%$ & \\
\hline & severe & 26 & $5 \%$ & 41 & $17 \%$ & \\
\hline Emotional problems & no & 242 & $49 \%$ & 119 & $49 \%$ & 0.935 \\
\hline & yes & 251 & $51 \%$ & 125 & $51 \%$ & \\
\hline Behaviour or psychological problem & no & 304 & $62 \%$ & 121 & $50 \%$ & ** \\
\hline & yes & 189 & $38 \%$ & 123 & $50 \%$ & \\
\hline
\end{tabular}

Notes: ${ }^{*} p<0.05 ;{ }^{* *} p<0.01 ;{ }^{* * *} p<0.001 ;$ CVD cardiovascular disease, CVA cerebrovascular accident, ICF International Classification of Functioning, Disability and Health

81 years. The average age of those who did and did not utilise HCBS was 81 years and 78 years, respectively. In addition, $62-65 \%$ of the patients were female, $52-58 \%$ had a spouse, and 59-65\% lived with their family. Compared with non-users, patients who used HCBS had significantly impaired physical and psychological functions. Finally, the use of services was significantly corelated to the ICF-based measurement of functioning, with $61 \%$ of users having mild to severe problems.

\section{Pattern of Service Utilisation}

Figure 1 presents a description of each service used. For 244 users, variance and mean (SD) of the frequency distribution were 0.350 and $1.28( \pm 0.592)$, respectively. The most frequently used services were assistive devices (39.1\%), followed by home services (18.9\%), adult day care (10.3\%), supportive care for caregivers (9.3\%), community care centres (4.8\%), transportation services (3.8\%), barrier-free environments $(3.2 \%)$, home nursing care $(2.9 \%)$, respite care $(2.2 \%)$, home meal delivery $(1.9 \%)$, home- and community-based rehabilitation $(1.3 \%)$, school of wisdom (1.3\%), mobile shower/bath services $(0.6 \%)$, and adult foster care $(0.3 \%)$. In total, this study found that $78 \%$ of patients utilised one service, $17 \%$ utilised two services at the same time, and $4 \%$ utilised three services, whereas only $1 \%$ utilised four services. Overall, the majority of patients with dementia tended to use one service, with only $20 \%$ using multiple services.

\section{Factors Influencing Service Utilisation}

Table 2 shows the factors that affected the use of services by patients with dementia $(n=737)$. Age was 


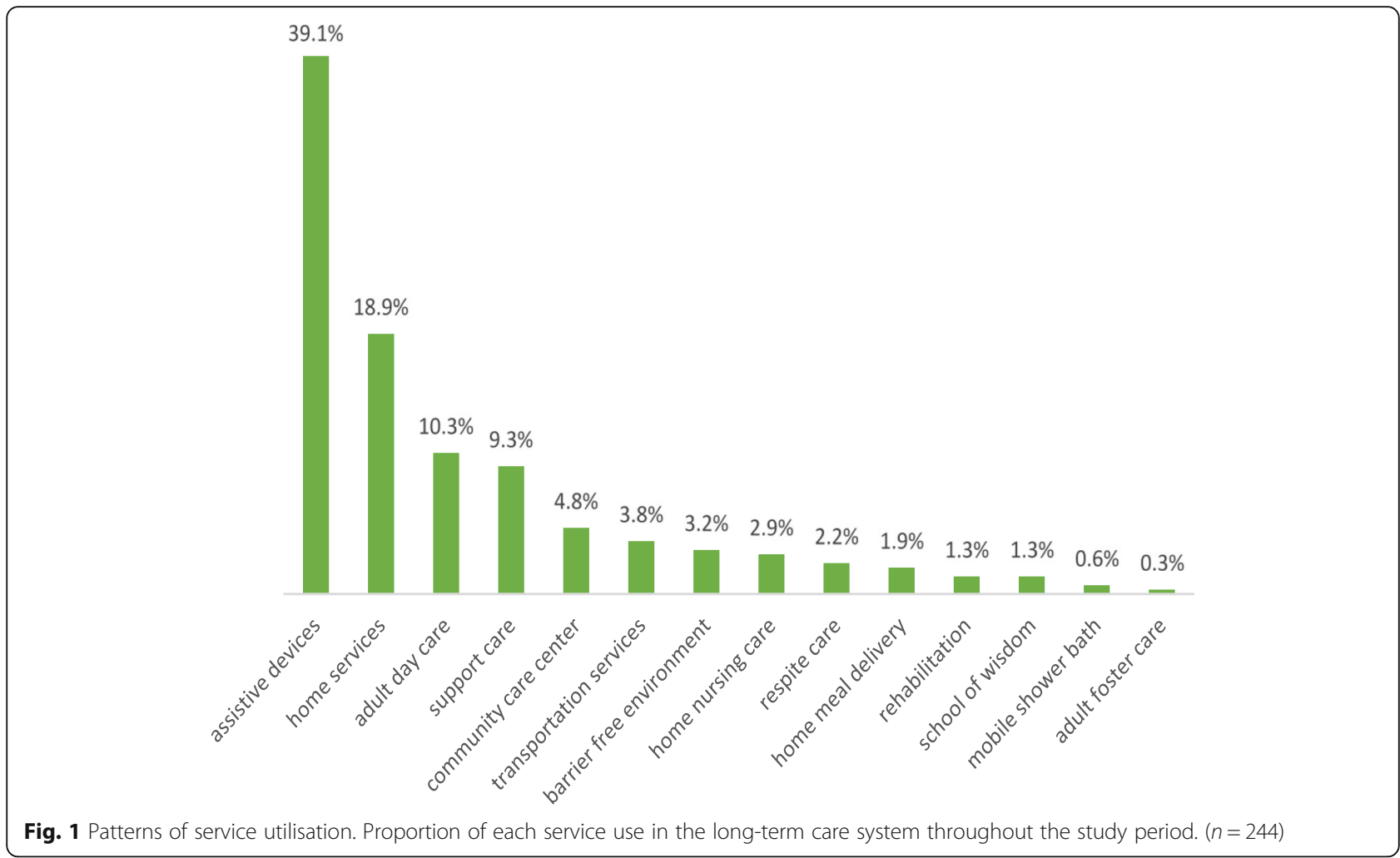

Table 2 Factors influencing HCBS utilisation as determined by generalised linear regression analyses $(N=737)$

\begin{tabular}{|c|c|c|c|c|c|c|c|c|}
\hline \multirow{3}{*}{ Age $(\leq 70)$} & \multirow[b]{3}{*}{$\geq 81$} & \multirow[t]{2}{*}{$\operatorname{Exp}(B)$} & & \multicolumn{3}{|c|}{$95 \% \mathrm{Cl}$} & & \multirow[t]{2}{*}{$p$ value } \\
\hline & & & & \multicolumn{2}{|l|}{ Lower } & \multicolumn{2}{|l|}{ Upper } & \\
\hline & & 1.83 & ( & 1.09 & - & 3.05 & ) & * \\
\hline & $71-80$ & 1.69 & ( & 1.03 & - & 2.78 & ) & * \\
\hline Sex (female) & male & 1.02 & ( & 0.73 & - & 1.43 & ) & 0.912 \\
\hline Marital status (married) & unmarried & 0.75 & ( & 0.53 & - & 1.08 & ) & 0.121 \\
\hline \multirow{4}{*}{$\begin{array}{l}\text { Main caregiver } \\
\text { (myself) }\end{array}$} & foreign worker & 0.67 & ( & 0.36 & - & 1.25 & ) & 0.204 \\
\hline & relatives/friends & 1.02 & ( & 0.53 & - & 1.97 & ) & 0.946 \\
\hline & children & 0.87 & ( & 0.48 & - & 1.56 & ) & 0.638 \\
\hline & spouse/partner & 0.56 & ( & 0.31 & - & 1.00 & ) & 0.051 \\
\hline Hypnotics (no) & yes & 1.10 & ( & 0.79 & - & 1.53 & ) & 0.587 \\
\hline CVD (no) & yes & 1.27 & ( & 0.83 & - & 1.93 & ) & 0.268 \\
\hline CVA (no) & yes & 1.46 & ( & 0.97 & - & 2.20 & ) & 0.068 \\
\hline \multirow{3}{*}{$\begin{array}{l}\text { Dependency status } \\
\text { (without ICF problems) }\end{array}$} & severe & 3.18 & ( & 2.00 & - & 5.05 & ) & $* * *$ \\
\hline & moderate & 2.62 & ( & 1.67 & - & 4.10 & ) & $* * *$ \\
\hline & mild & 1.69 & ( & 1.15 & - & 2.49 & ) & $* *$ \\
\hline Emotional problems (no) & yes & 0.84 & ( & 0.59 & - & 1.18 & ) & 0.314 \\
\hline $\begin{array}{l}\text { Behaviour or psychological } \\
\text { problem (no) }\end{array}$ & yes & 1.22 & ( & 0.88 & - & 1.70 & ) & 0.233 \\
\hline
\end{tabular}

Notes: ${ }^{*} p<0.05 ;{ }^{* *}<0.01 ;{ }^{* * *} p<0.001 ; C l$ confidence interval; CVD cardiovascular disease, CVA cerebrovascular accident, ICF International Classification of Functioning, Disability and Health 


\section{A. Top 5 HCBS use - with or without ICF problem}

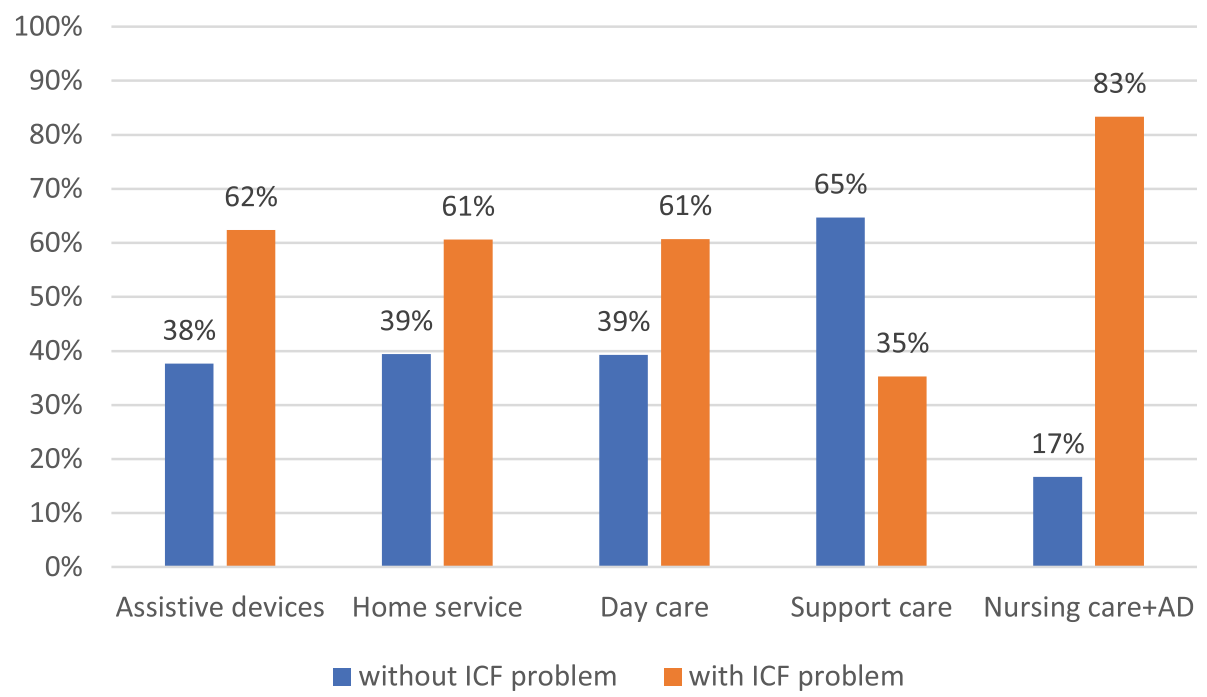

\section{B. Top 5 HCBS use-with ICF problem}

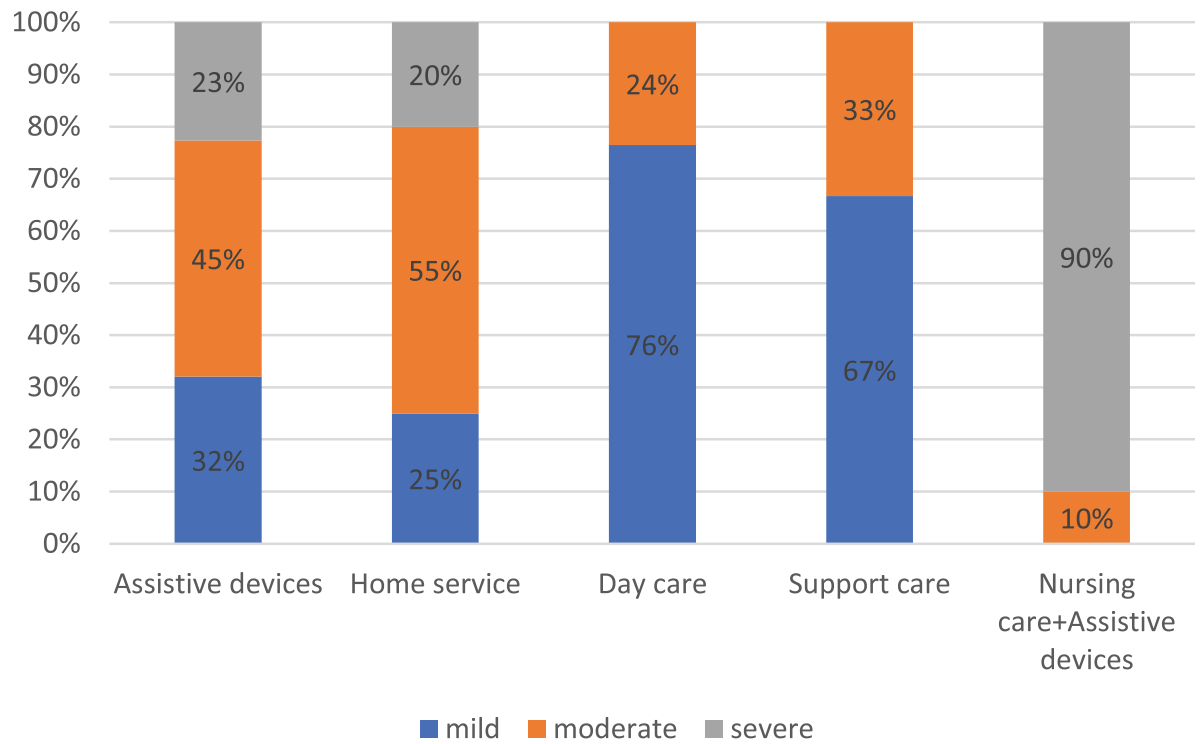

Fig. 2 a. Percentage of participants with ICF-related or non-ICF related problems by the top 5 home- and community-based services used. $\mathbf{b}$. Percentage of service utilisation by dementia-related top 5 HCBS used by participants with mild, moderate and severe ICF problems. (ICF: International Classification of Functioning, Disability and Health)

positively correlated with the number of services used, as patients who were older used more services. The adjusted risk ratio (RR) of service ranged from $1.69(95 \%$ $\mathrm{CI}=1.03-2.78)$ in patients aged $71-80$ to 1.83 (95\% $\mathrm{CI}=1.09-3.05)$ in patients aged $\geqq 81$. The number of services used by patients with dementia increased with the severity of dependence. Compared with patients without problems according to the ICF-based assessment, the adjusted RR of service use ranged from 1.69 , in patients with mild dependency, to 2.62 , in those with moderate dependency, and to 3.18 , in patients with severe dependency. These results imply that the most important factor influencing service usage is dependency status.

\section{Services used by Dependency Status}

Figure 2a-b displays the percentage of dementia-related dependency status by the top 5 HCBS utilisation 
categories (assistive devices, home service, day care, support care, nursing care and assistive devices). In most types of HCBS, the majority of services used were attributed to ICF problems. This was especially relevant in the case of assistive devices, which were used mainly by patients with moderate disability ( $45 \%)$, followed by mild disability (32\%), and severe disability (23\%). In addition, people with mild dependency used more day care services $(76 \%)$ and support care $(67 \%)$ than those with moderate dependency, the majority of whom used more home services $(55 \%)$, and those with severe dependency, that mainly used assistive devices in combination with nursing care $(90 \%)$. Accordingly, patients with dementia with mild or no problems according to ICF-based assessment were more likely to use community support services, whereas those with moderate to severe dependency were more likely to use in-home care services.

\section{Discussion}

The purpose of this study was to examine patterns of HCBS usage among community-dwelling patients with established dementia diagnoses. The rate of community services used by people with dementia was not as high as expected (33\%), and $78 \%$ of users only used one service, mainly assistive devices. Dementia with disability was the main determinant of most utilisation. The severity of dependency affects the use of services, implying that more service resources may be used by patients with dementia with concomitant severe dependency. This research has made an important contribution to our understanding of the use of care support service systems for patients with dementia residing in the community.

Ageing populations with a growing incidence of disabilities and dementia are important factors driving the increased demand for LTC. LTC 1.0 compiled policies mainly designed for disabled people, whereas services for people with mild to moderate dementia were lacking. Therefore, LTC 2.0 expanded services for people with dementia to eliminate this service gap. Based on the $8 \%$ prevalence rate of dementia in Taiwan, more than 260, 000 people suffer from dementia; however, only $30 \%$ of them are properly diagnosed and treated accordingly [21]. This study shows that the utilisation rate of LTC services for the elderly with dementia was $33 \%$. Since the goal of the LTC plan is to build a powerful and accessible service system, the coverage is clearly insufficient. The dementia services provided by LTC are limited to patients who have been diagnosed with dementia [22], resulting in unavailability of needed care to people who have not yet been diagnosed with dementia but have cognitive impairment. This raises the concern about whether there is adequate care for people with early dementia and how to implement the connection services such as subacute care for those with cognitive decline at an early stage.

Supportive services such as assistive technology (AT) devices have been shown to be associated with the time that people with dementia remain in their own homes [23, 24]. The establishment of 10 AT centres in the Changhua area has not only increased the convenience of accessing AT services but has also enhanced the public's willingness to use AT devices. A recent review found that dementia caregivers find certain types of AT useful, such as GPS trackers, motion sensors, or medication reminders [25]. This trend may reflect changes in basic health conditions (for example, a decrease in the level of disability) and a tendency to shift to the use of equipment rather than human care (i.e., increasing acceptance of technology). It is well known that nearly $50 \%$ of users accept AT devices, and those with moderate to severe dependency had the highest rate of AT use. However, it is not clear whether providing AT alone can maintain the independence of patients or what the actual impact on caregiver burden and satisfaction is. Therefore, in the future it is necessary to address if patients who are highly dependent on AT equipment require other inputs or options.

It is not surprising that dependency status is related to increased LTC utilisation [26]. Dementia is related to age, and even after extensive control for age, disability remains an important driver of LTC use. Interestingly, the use of service resources varies according to the level of dependency. People with mild dementia tend to prefer to use community support services (day care centres), whereas those with moderate to severe dependency tend to prefer to use in-home care services (home care and nursing care). This raises the concern about whether there is adequate care for people with dementia at different levels of disability. The degree of dependence may affect a person's living arrangements, which in turn may affect choices related to the use of LTC services [27]. A recent study has shown that about $75 \%$ of dementia patients in Western countries are cared for by their family members [28]. Older people with dementia need much more support and protection from their family or society, so older individuals with higher dependency levels are more likely to live with a spouse or adult children. Hence, informing family caregivers' about the early symptoms of the disease and the existing service resources is essential to facilitate early diagnosis of impaired cognitive function and provide the required services in time.

In contrast to national data on service use in Taiwan [29], home care was not the most commonly used service in this study, and the use of home care was much greater among those with moderate disability. It could 
be expected that when functional disability is more severe, such as difficulty in walking and eating, the frequency of in-home help usage will be substantially greater. The needs of people with dementia and their caregivers are diverse, and the services that support them do not always meet their needs effectively [30]. Very few studies have been performed on the feelings of dementia caregivers about receiving home services and whether or not their needs are met, and the effect of home services in reducing the burden of caregivers is not yet clear [31, 32]. Accordingly, the expression of care needs and whether the care needs are being met are important indicators that affect LTC service use and are worthy of further discussion.

National LTC policies in different countries are influenced by the specific historic background, politics, resources, culture, and the role of the government in social welfare $[3,33,34]$. The different policies about care provision across countries vary in the way informal care is treated when care needs assessment for publicly funded LTC. For example, in England and Australia, the amount of informal care provided by relatives is taken into account and financed by the government, whereas this is not the case in France or Japan [35]. LTC 2.0 considers the unaffordability of private care (for example, for low-income households) and the unavailability of informal care (for example, for people without family in close proximity), and establishes protocols to meet people's needs. The current policies are designed to encourage that the elderly stay at their own home for as much time as possible, and therefore, most resources have been allocated to in-home care and community care. Hence, the purpose of HCBS is to supplement the insufficiency of informal care and to support the elderly with community-living or low-income and able to ageing in place [8]. Although the Taiwan policies are distinctly 'Taiwanese', all countries worldwide are facing similar challenges now and will need to develop more comprehensive strategies to address this multidimensional challenge.

This study examined a cohort of community-dwelling older adults with dementia using comprehensive telephone interview data from a medical centre in central Taiwan. However, this study had some limitations. As we did not capture the same service during the 2 years of follow-up, the impact of dementia on service utilisation has been underestimated. For example, people who used service A at baseline could use services A and B in the second year, but we only counted the use of two services. Although we could provide a snapshot of the use of dementia-related community services in our cohort, we lacked information on their degree of dementia. As the cognition and physical function of patients degenerate simultaneously during the tracking process, ICF- based assessment of problems was used instead of the degree of dementia as the main distinguishing criterion. In addition, in this research, only the opinions of those using HCBS were collected, whereas the opinions of service providers or those who did not use services were not obtained. Therefore, future research should focus on the views of those who do not use services and compare them with those who do use services. Finally, the dataset was obtained from a medical centre in central Taiwan, and the sample size focused only on those with dementia, diagnosed using a cross-sectional study design; therefore, generalisation of the results should be performed with caution under the LTC policy nationwide.

\section{Conclusions}

This study uses Taiwan as an example to explore the experience of LTC service use. Recognising the diversity of elderly people with dementia, at the level of functional impairment and in their ability to secure help without LTC support, is essential for developing policies that meet the wide range of needs of elderly people. In addition to providing in-home care or community support services based on the degree of dementia and disability, further research should be conducted on the benefits of AT devices. Increasing awareness of service availability is essential to enable caregivers to better match service use with patient needs. To ensure that recipients' needs are met after enrolling in the publicly funded LTC system, a more accurate protocol to assess the patient's needs must be developed and implemented policies should encourage all types of service resources to settle in areas currently not covered. This will ensure recipients continue to live in their home communities and receive care, which will achieve the goal of the ageing in place policy.

\section{Abbreviations}

HCBS: Home- and community-based service; LTC: Long-term care; CCH: Changhua Christian Hospital; DSM-V: Diagnostic and Statistical Manual of Mental Disorders; ICF: International Classification of Functioning; AT: Assistive technology; GPS: Global Positioning System

\section{Acknowledgements}

We would like also to thank data collectors and study participants.

\section{Authors' contributions}

WFW, KMJ, and CMC designed the study. WFW and KMJ were involved in the data collection and supervision. CMC and YYS completed data processing and analysis. CMC, YYS, and KMJ interpreted the data and drafted the manuscript. All authors have critically reviewed the manuscript and read and approved the final manuscript.

\section{Funding}

Dementia research was supported by grants from the Changhua Christian Hospital (project no. 108-CCH-PRJ-174, 109-CCH-IPR-038). The funders had no role in the study design, data collection and analysis, decision to publish, or preparation of the manuscript. 


\section{Availability of data and materials}

The data that support the findings of this study are available from the dementia centre of Changhua Christian Hospital, but restrictions apply to the availability of these data, which were used under licence for the current study, and so are not publicly available. However, data are available from the authors upon reasonable request and with permission from the dementia centre of Changhua Christian Hospital.

\section{Declarations}

\section{Ethics approval and consent to participate}

The study was carried out in accordance with the Declaration of Helsinki and was approved by the Ethics Committee of Changhua Christian Hospital, Taiwan (approval no. 160615; 200128). Informed consent was not obtained from the patients because it was not requested for this retrospective study. Data were collected from electronic medical records and the data used were anonymous. No any administrative permission was required to access and use the medical records described in the study.

\section{Consent for publication}

Not applicable.

\section{Competing interests}

The authors declare that they have no conflict of interest.

\section{Author details}

'Department of Neurology, Changhua Christian Hospital, Changhua, Taiwan. 2Department of Holistic Wellness, Ming Dao University, Changhua, Taiwan. ${ }^{3}$ Department of Long Term Care, National Quemoy University, Kinmen, Taiwan. ${ }^{4}$ Big Data Center, Changhua Christian Hospital, No. 135, Nanhsiao Street, 500-06 Changhua City, Changhua County, Taiwan.

Received: 21 July 2020 Accepted: 19 April 2021

Published online: 01 May 2021

\section{References}

1. Chen CF, Fu TH. Policies and Transformation of Long-Term Care System in Taiwan. Ann Geriatr Med Res. 2020;24(3):187-94. https://doi.org/10.4235/a gmr.20.0038

2. Taiwan Alzheimer's Disease Association: Epidemiological survey of dementia. In:Taiwan, R.O.C. April 4. 2020. http://www.tada2002.org.tw/A bout/IsntDementia May 6, 2020.

3. World Health Organization. Key Policy Issues in Long-term Care. In. 2003.

4. Joshua L: Aging and Long Term Care Systems: A Review of Finance and Governance Arrangements in Europe, North America and Asia-Pacific. In Edited by Group WB. Washington, USA.; 2017.

5. Yang CC, Hsueh JY, Wei CY: Current Status of Long-term Care in Taiwan: Transition of Long-term Care Plan From 1.0 to 2.0. Int J Health Policy Manag 2020, 9(8):363-364. https://doi.org/10.15171/ijhpm.2019.115.

6. Hsueh J-Y, Wei C-Y, Yang C-C: Current Status of Long-term Care in Taiwan: Transition of Long-term Care Plan From 1.0 to 2.0. IJHPM 2020:1-2. https:// doi.org/10.15171/IJHPM.2019.115

7. National Quality Forum: Priority Setting for Healthcare Performance Measurement: Addressing Performance Measure Gaps for Dementia, Including Alzheimer's Disease-Final Report. In:Washington DC. 2014 http:// www.qualityforum.org/priority_setting_for_healthcare_performance_mea surement_alzheimers_disease.aspx May 1, 2020.

8. Kane R. Thirty Years of Home- and Community-Based Services: Getting Closer and Closer to Home. Generations (San Francisco Calif). 2012;36:6-13.

9. Wysocki A, Butler M, Kane RL, Kane RA, Shippee T, Sainfort F. Long-Term Services and Supports for Older Adults: A Review of Home and CommunityBased Services Versus Institutional Care. J Aging Soc Policy. 2015;27(3):25579. https://doi.org/10.1080/08959420.2015.1024545.

10. Chen Y-M, Berkowitz B. Older adults' home- and community-based care service use and residential transitions: a longitudinal study. BMC Geriatrics. 2012;12(1):44. https://doi.org/10.1186/1471-2318-12-44.

11. Bieber A, Nguyen N, Meyer G, Stephan A. Influences on the access to and use of formal community care by people with dementia and their informal caregivers: a scoping review. BMC Health Serv Res. 2019;19(1):88. https://doi. org/10.1186/s12913-018-3825-z.
12. Stephan A, Bieber A, Hopper L, Joyce R, Irving K, Zanetti O, Portolani E, Kerpershoek L, Verhey F, de Vugt $M$, et al. Barriers and facilitators to the access to and use of formal dementia care: findings of a focus group study with people with dementia, informal carers and health and social care professionals in eight European countries. BMC Geriatr. 2018;18(1):131-1. https://doi.org/10.1186/s12877-018-0816-1.

13. Schaller S, Mauskopf J, Kriza C, Wahlster P, Kolominsky-Rabas PL. The main cost drivers in dementia: a systematic review. Int J Geriatr Psychiatry. 2015 30(2):111-29. https://doi.org/10.1002/gps.4198.

14. Griffith LE, Gruneir A, Fisher K, Panjwani D, Gandhi S, Sheng L, Gafni A, Patterson C, Markle-Reid M, Ploeg J. Patterns of health service use in community living older adults with dementia and comorbid conditions: a population-based retrospective cohort study in Ontario, Canada. BMC Geriatrics. 2016;16(1):177. https://doi.org/10.1186/s12877-016-0351-x.

15. American Psychiatric Association. Diagnostic And Statistical Manual Of Mental Disorders(5th ed.; DSM-5). Washington, DC; 2013.

16. Jhang KM, Chang MC, Lo TY, Lin CW, Wang WF, Wu HH. Using The Aprior Algorithm To Classify The Care Needs Of Patients With Different Types Of Dementia. Patient Prefer Adherence. 2019:13:1899-912. https://doi.org/1 0.2147/ppa.S223816

17. Stucki G, Kostanjsek N, Ustun B, Cieza A. ICF-based classification and measurement of functioning. Eur J Phys Rehabil Med. 2008;44(3):315-28.

18. Frome EL. The Analysis of Rates Using Poisson Regression Models. Biometrics. 1983;39(3):665-74. https://doi.org/10.2307/2531094.

19. Coxe S, West SG, Aiken LS. The analysis of count data: a gentle introduction to poisson regression and its alternatives. J Pers Assess. 2009;91 (2):121-36. https://doi.org/10.1080/00223890802634175.

20. Koletsi D, Pandis N. Poisson regression. Am J Orthod Dentofacial Orthop. 2017;152(2):284-5. https://doi.org/10.1016/j.ajodo.2017.05.009.

21. Ministry of Health and Welfare. Dementia Friendly Taiwan by 2025 (in (Chinese). In:Taiwan, R.O.C. 2018

22. Ministry of Health and Welfare. 2017 Taiwan Health and Welfare Report. In:R O.C. Taiwan. 2017 https://www.mohw.gov.tw/cp-137-40301-2.html.

23. Meiland F, Innes A, Mountain G, Robinson L, van der Roest $H$, García-Casal JA, Gove D, Thyrian JR, Evans S, Dröes R-M, et al. Technologies to Support Community-Dwelling Persons With Dementia: A Position Paper on Issues Regarding Development, Usability, Effectiveness and Cost-Effectiveness, Deployment, and Ethics. JMIR Rehabil Assist Technol. 2017;4(1):e1-1. https:// doi.org/10.2196/rehab.6376.

24. Brims L, Oliver K. Effectiveness of assistive technology in improving the safety of people with dementia: a systematic review and meta-analysis. Aging Ment Health. 2019;23(8):942-51. https://doi.org/10.1080/13607863.201 8.1455805.

25. Sriram V, Jenkinson C, Peters M. Informal carers' experience of assistive technology use in dementia care at home: a systematic review. BMC Geriatrics. 2019;19(1):160. https://doi.org/10.1186/s12877019-1169-0.

26. de Meijer CA, Koopmanschap MA, Koolman XH, van Doorslaer EK. The role of disability in explaining long-term care utilization. Med Care. 2009;47(11): 1156-63. https://doi.org/10.1097/MLR.0b013e3181b69fa8.

27. Zhang $L$, Zeng $Y$, Fang $Y$. The effect of health status and living arrangements on long term care models among older Chinese: A crosssectional study. PloS one. 2017;12(9):e0182219-9. https://doi.org/10.1371/ journal.pone.0182219.

28. Etters L, Goodall D, Harrison BE. Caregiver burden among dementia patient caregivers: a review of the literature. J Am Acad Nurse Pract. 2008;20(8):423-8. https://doi.org/10.1111/j.1745-7599.2008.00342.x.

29. Ministry of Health and Welfare: National Ten-year Long-term Care Plan 2.0 2017 Years to 2026 Years (In Chinese). In:Taiwan, R.O.C. 2016 https://www. mohw.gov.tw/dl-46355-2d5102fb-23c8-49c8-9462-c4bfeb376d92.html (accessed 12 June 2020).

30. Morrisby C, Joosten A, Ciccarelli M. Do services meet the needs of people with dementia and carers living in the community? A scoping review of the international literature. Int Psychogeriatr. 2018;30(1):5-14. https://doi.org/1 $0.1017 /$ s1041610217001491.

31. Olazarán RJ, Sastre Paz M, Martín Sánchez S: Health care in dementia: Satisfaction and needs of the caregiver. Neurología (English Edition) 2012, 27(4):189-196. https://doi.org/10.1016/j.nrleng.2011.07.001.

32. Liu LF, Wang WM, Chen YJ. The Effectiveness of Home Services in Taiwan: A People-Centered Approach. Int J Environ Res Public Health. 2018:15(11): 2605. https://doi.org/10.3390/ijerph15112605. 
33. Karlsson M, Mayhew L, Plumb R, Rickayzen B: An International Comparison of Long-Term Care Arrangements. An Investigation into the Equity,Efficiency \& Sustainability of the Long-term Care Systems in Germany,Japan,Sweden,the U.K. \& the U.S.A. London, United Kingdom: Faculty of Actuarial Science and Statistics,Cass Business School; 2004.

34. Elliott S, Golds S, Sissons I, Wilson H. Long-term care: a review of global funding models. British Actuarial Journal. 2015;20(1):167-208. https://doi. org/10.1017/S1357321714000300.

35. Robertson R, Gregory S, Jabbal J: The social care and health systems of nine countries: The King's Fund.; 2014.

\section{Publisher's Note}

Springer Nature remains neutral with regard to jurisdictional claims in published maps and institutional affiliations.

Ready to submit your research? Choose BMC and benefit from:

- fast, convenient online submission

- thorough peer review by experienced researchers in your field

- rapid publication on acceptance

- support for research data, including large and complex data types

- gold Open Access which fosters wider collaboration and increased citations

- maximum visibility for your research: over $100 \mathrm{M}$ website views per year

At BMC, research is always in progress.

Learn more biomedcentral.com/submissions 